\title{
TITLE:
}

\section{Defect energetics in ZnO: A hybrid Hartree-Fock density functional study}

\section{$\operatorname{AUTHOR}(\mathrm{S})$ :}

Oba, Fumiyasu; Togo, Atsushi; Tanaka, Isao; Paier, Joachim; Kresse, Georg

\section{CITATION:}

Oba, Fumiyasu ...[et al]. Defect energetics in ZnO: A hybrid Hartree-Fock density functional study. PHYSICAL REVIEW B 2008, 77(24): 245202.

\section{ISSUE DATE:}

2008-06

URL:

http://hdl.handle.net/2433/84635

RIGHT:

(C) 2008 The American Physical Society 
PHYSICAL REVIEW B 77, 245202 (2008)

\title{
Defect energetics in ZnO: A hybrid Hartree-Fock density functional study
}

\author{
Fumiyasu Oba, ${ }^{*}$ Atsushi Togo, and Isao Tanaka \\ Department of Materials Science and Engineering, Kyoto University, Sakyo, Kyoto 606-8501, Japan \\ Joachim Paier and Georg Kresse \\ Faculty of Physics, University of Vienna and Center for Computational Materials Science, A-1090 Wien, Austria
}

(Received 1 April 2008; published 9 June 2008)

\begin{abstract}
First-principles calculations based on hybrid Hartree-Fock density functionals provide a clear picture of the defect energetics and electronic structure in $\mathrm{ZnO}$. Among the donorlike defects, the oxygen vacancy and hydrogen impurity, which are deep and shallow donors, respectively, are likely to form with a substantial concentration in $n$-type $\mathrm{ZnO}$. The zinc interstitial and zinc antisite, which are both shallow donors, are energetically much less favorable. A strong preference for the oxygen vacancy and hydrogen impurity over the acceptorlike zinc vacancy is found under oxygen-poor conditions, suggesting that the oxygen vacancy contributes to nonstoichiometry and that hydrogen acts as a donor, both of which are without significant compensation by the zinc vacancy. The present results show consistency with the relevant experimental observations.
\end{abstract}

DOI: 10.1103/PhysRevB.77.245202

PACS number(s): 61.72.J-, 61.72.Bb, 71.55.Gs

\section{INTRODUCTION}

With outstanding versatility in electrical, optical, and chemical properties, $\mathrm{ZnO}$ is recognized as a key material in a wide variety of commercial applications. Renewed interest has recently emerged for its ultraviolet light emission capabilities. ${ }^{1}$ Despite the fact that a fair amount of fundamental research was stimulated by its technological importance, the defect that is relevant to the $n$-type conductivity of undoped $\mathrm{ZnO}$ remains controversial. In the last decade, firstprinciples studies have provided insight into the defect energetics and electronic structure. ${ }^{2-17}$ The results rather consistently indicate that the oxygen vacancy $\left(V_{\mathrm{O}}\right)$ has the lowest formation energy among the donorlike defects but forms a deep electronic state. The zinc interstitial $\left(\mathrm{Zn}_{i}\right)$ can be a shallow donor but it has high formation energy. Whereas the native defects are, thus, unlikely to be the major source of the $n$-type conductivity, the hydrogen impurity was suggested as a background shallow donor, ${ }^{3,11}$ which is followed by an experimental support. ${ }^{18-21}$ An optically excited metastable configuration of $V_{\mathrm{O}}$ with a shallow donor state ${ }^{7}$ and the complex of $\mathrm{Zn}_{i}$ and the nitrogen impurity ${ }^{22}$ were also proposed as candidates.

The theoretically derived defect energetics, however, possesses huge uncertainties due to the inaccurate description of the band structure of $\mathrm{ZnO}$ in using the local density functionals and the exceedingly slow energy convergence with the supercell size. Notably, the local density approximation (LDA) and generalized gradient approximation (GGA) yield significantly underestimated band gaps, i.e., $0.7-0.8 \mathrm{eV}$, compared to the experimental value of $3.44 \mathrm{eV}^{23}$ Two ad hoc correction schemes, i.e., LDA $+U$ and empirical a posteriori band-gap corrections, have been used. Unfortunately, the resultant energetics even qualitatively depends on the correction procedures. For instance, the $V_{\mathrm{O}}$ formation energy is reported to be between 0.8 and $3.7 \mathrm{eV}$, even using the same plane-wave code but different correction schemes. ${ }^{7-11,14,15}$ The situation is largely discomforting. Hence, a concise treatment that allows for large supercells and accurate band gaps is urgently needed. Hybrid Hartree-Fock density func- tionals seem to offer such a solution but the yet presented calculations are limited to small supercells without finite-size corrections ${ }^{16}$ or to an embedded cluster approach that does not describe the delocalized conduction-band states well. ${ }^{17}$

In this paper, we, thus, report a hybrid functional study on the defects in $\mathrm{ZnO}$, which is combined with careful finitesize corrections. The results are discussed with a focus on the energetics and electronic structure of the donorlike defects.

\section{COMPUTATIONAL METHODS}

The calculations were performed using the planewave projector augmented-wave (PAW) method ${ }^{24}$ with the Perdew-Burke-Ernzerhof (PBE) GGA functional, ${ }^{25}$ and the PBE0,${ }^{26}$ and Heyd-Scuseria-Ernzerhof (HSE) ${ }^{27}$ hybrid functionals as implemented in the VASP code. ${ }^{28-30}$ The screening parameter in HSE was fixed at a value of $0.2 \AA^{-1} .{ }^{27}$ The implementation issues and extensive tests of these hybrid functionals have been reported elsewhere. ${ }^{31,32}$ The PAW data sets with radial cutoffs of $1.2,1.0$, and $0.6 \AA$ for $\mathrm{Zn}, \mathrm{O}$, and $\mathrm{H}$, respectively, were employed with a plane-wave cutoff energy of $300 \mathrm{eV}$, where $\mathrm{Zn} 3 d, 4 s, 4 p, \mathrm{O} 2 s, 2 p$, and $\mathrm{H} 1 s$ electrons were described as valence electrons. For the native defects, $V_{\mathrm{O}}, \mathrm{Zn}_{i}$ at the octahedral and tetrahedral sites $\left[\mathrm{Zn}_{i(o)}\right.$ and $\left.\mathrm{Zn}_{i(t)}\right]$, the zinc antisite $\left(\mathrm{Zn}_{\mathrm{O}}\right)$, and the zinc vacancy $\left(V_{\mathrm{Zn}}\right)$, as a representative acceptorlike defect, ${ }^{2,4-6,8,10,14,15,17}$ were considered. Regarding the hydrogen impurity, a number of different forms of shallow donors have been experimentally observed. ${ }^{18-21}$ First-principles studies suggested the presence of several stable/metastable sites for isolated interstitial hydrogen $\mathrm{n}^{3,12}$ and its mobile nature, implying a trap by other defects. ${ }^{13}$ Furthermore, hydrogen was predicted to occupy the substitutional oxygen site. ${ }^{11}$ Among the forms proposed, we considered two characteristic cases: interstitial hydrogen at an energetically favorable $\mathrm{Zn}-\mathrm{O}$ bond-center site along the $c$ axis $\left(\mathrm{H}_{i}\right)^{3,12}$ and substitutional hydrogen at the oxygen site $\left(\mathrm{H}_{\mathrm{O}}\right)$.

For the defect calculations using the hybrid functionals, 192 -atom cells and a $\Gamma$-only $k$-point sampling were em- 
ployed. The large cells were chosen to minimize the errors in the finite-size corrections of formation energies, as will be mentioned later. The $\Gamma$-only sampling, however, was found to cause relatively large errors in the formation energies of $V_{\mathrm{O}}$ in the neutral and + charge states (denoted also as $V_{\mathrm{O}}^{0}$ and $V_{\mathrm{O}}^{+}$hereafter, where the superscripts designate the charge states), and $\mathrm{Zn}_{\mathrm{O}}$ in the $0-3+$ charge states, i.e., $0.1-0.2 \mathrm{eV}$, owing to the selective sampling of the bottom of the defectinduced localized band with a slight dispersion. The errors were corrected using the difference between the average of the eigenvalues of the defect-induced band and the eigenvalue at the $\Gamma$ point. Systematic test calculations indicate that after the corrections, the errors in the formation and transition energies are small for all the considered defects, i.e., $\sim 0.1$ and $\sim 0.05 \mathrm{eV}$, respectively. With the lattice constants fixed at the optimized values for the perfect crystal, the internal coordinates in the defect supercells were relaxed to reduce the residual forces to less than $0.05 \mathrm{eV} / \AA$. Spin polarization was taken into account for $V_{\mathrm{O}}^{+}, \mathrm{Zn}_{\mathrm{O}}^{3+}$, and $V_{\mathrm{Zn}}$, which form half-occupied localized states.

The defect formation energy was evaluated as ${ }^{33}$

$$
E_{f}=E_{t}^{d}-E_{t}^{p}-\sum_{i} \Delta n_{i} \mu_{i}+q E_{\mathrm{F}}
$$

where $E_{t}^{d}$ and $E_{t}^{p}$ denote the total energies of the supercell containing a defect with the charge $q$ and the perfect-crystal supercell, and $\Delta n_{i}$ is the difference in the number of constituent atoms of type $i . \mu_{i}$ and $E_{\mathrm{F}}$ are the atomic chemical potential and the Fermi energy, respectively. $\mu_{\mathrm{Zn}}$ and $\mu_{\mathrm{O}}$ were assumed to vary between the oxygen-poor (zinc-rich) limit $\left[\mu_{\mathrm{Zn}}=\mu_{\mathrm{Zn} \text { (bulk) }}\right.$ and $\mu_{\mathrm{O}}=1 / 2 \mu_{\mathrm{O}_{2}}+\Delta H_{f}$, where $\Delta H_{f}$ denotes the heat of formation of $\mathrm{ZnO}]$ and the oxygen-rich limit $\left[\mu_{\mathrm{Zn}}=\mu_{\mathrm{Zn}(\text { bulk })}+\Delta H_{f}\right.$ and $\left.\mu_{\mathrm{O}}=1 / 2 \mu_{\mathrm{O}_{2}}\right]$. For hydrogen, $\mu_{\mathrm{H}}$ $=1 / 2 \mu_{\mathrm{H}_{2}}$ was taken as the hydrogen-rich (oxygen-poor) limit. The calculated total energies of the $\mathrm{Zn}$ crystal, and the $\mathrm{O}_{2}$ and $\mathrm{H}_{2}$ molecules were used as the respective chemical potentials.

Errors in the defect formation energies due to the spurious electrostatic interactions in the finite-sized cells were corrected using the scheme proposed by Leslie and Gillan, ${ }^{34}$ and by Makov and Payne. ${ }^{35}$ It assumes that the interactions between the multipoles at the defect site, and those between the multipoles and the jellium background, lead to $L^{-1}, L^{-3}$, and $L^{-5}$ dependences of the formation energies (where $L$ is the average interdefect distance). The $L^{-1}$ term corresponds to the Madelung energy for an array of point charges $q_{c}$ in an effective medium with a static dielectric constant $\epsilon_{0}, E_{c}$ $=\alpha q_{c}^{2} / 2 \epsilon_{0} L$, where $\alpha$ is the appropriate Madelung constant. This term is considered to be dominant for the charged defects in the ionic crystals. ${ }^{34,35}$

First, the corrections for the Madelung energies were applied to the formation energies that were calculated by using the hybrid functionals. As will be detailed later, $q_{c}$ was determined on the basis of the characteristics of defect-induced electronic states instead of using the formal defect charge $q$. For $\epsilon_{0}$, an experimental value of 8.1 (Ref. 36) was taken. The formation energies after the Madelung energy corrections were then extrapolated to the dilute (infinite cell-size) limit
TABLE I. Calculated fundamental properties of $\mathrm{ZnO}$

\begin{tabular}{lccccc}
\hline \hline & $\begin{array}{c}a \\
(\AA)\end{array}$ & $\begin{array}{c}c \\
(\AA)\end{array}$ & $\begin{array}{c}\Delta H_{f} \\
(\mathrm{eV})^{\mathrm{a}}\end{array}$ & $\begin{array}{c}E_{3 d} \\
(\mathrm{eV})\end{array}$ & $\begin{array}{c}E_{g} \\
(\mathrm{eV})\end{array}$ \\
\hline GGA-PBE & 3.286 & 5.299 & -2.82 & -4.8 & 0.74 \\
PBE0 & 3.257 & 5.223 & -3.03 & -5.9 & 3.18 \\
HSE & 3.261 & 5.225 & -3.01 & -5.8 & 2.49 \\
HSE $(a=0.375)$ & 3.249 & 5.196 & -3.13 & -6.4 & 3.43 \\
Experiment $^{\mathrm{b}}$ & 3.242 & 5.188 & -3.63 & -7.5 & 3.44 \\
\hline
\end{tabular}

a Errors in the binding energy of the $\mathrm{O}_{2}$ molecule associated with the large PAW radial cutoff were corrected using the binding energy calculated with a smaller radial cutoff of $0.6 \AA$.

${ }^{\mathrm{b}}$ References 23 and 39-41.

using $L^{-3}$ dependences derived from systematic calculations with the GGA, which were scaled by the ratio of the GGAestimated $\epsilon_{0}$ to the experimental value. The large 192-atom cells employed in the hybrid functional calculations were confirmed to produce small errors in the extrapolation: The mean (maximum) error was estimated to be $0.02(0.06) \mathrm{eV}$. The $L^{-5}$ dependent contributions, which are expected to be sufficiently small for the large cells, were not considered.

The GGA calculations for the evaluation of the $L^{-3}$ dependences were performed using seven supercells with increasing size, containing up to 784 atoms, and $k$-point sets corresponding to $8 \times 8 \times 6$ or denser meshes for the primitive cell. A correction for filling the conduction band or emptying the valence band $^{33,37}$ was considered.

\section{RESULTS AND DISCUSSION}

The fundamental properties of $\mathrm{ZnO}$ that were calculated by using the hybrid functionals are summarized in Table I, alongside those with the GGA and experimental values. When compared to the GGA, the PBE0 and HSE hybrid functionals improve not only the band gap $\left(E_{g}\right)$ and the $\mathrm{Zn} 3 d$ position $\left(E_{3 d}\right)$ but also the heat of formation $\left(\Delta H_{f}\right)$ and the lattice constants, as recently reported for HSE. ${ }^{38}$ However, the underestimation of $E_{g}$ and the underbinding of $\mathrm{Zn} 3 d$ still prevail. This is particularly obvious for HSE, yielding a gap of $2.49 \mathrm{eV}$. For the hybrid functionals, the optimal amount of the nonlocal Fock-exchange can be system dependent. ${ }^{26}$ By increasing the fraction from the original value of $a=0.25$ to 0.375 within the formalism of HSE, $E_{g}$ and $E_{3 d}$ approach the experimental values. At the same time, the lattice constants $\left(a\right.$ and $c$ ) and $\Delta H_{f}$ are reproduced best, which is an indispensable requirement for predicting accurate defect energies. The defect calculations were, therefore, mainly performed using HSE with the fraction of the nonlocal exchange fixed at $a=0.375$. HSE shows much faster convergence of the exchange energy with respect to the number of $k$ points than PBE0 not only for metals but also for semiconductors and insulators, ${ }^{32}$ which is advantageous in largescale hybrid functional calculations, as performed in the present study. However, since the approach with an increased fraction of the nonlocal exchange is semiempirical, selected calculations were also performed using PBE0 to confirm the main results. 

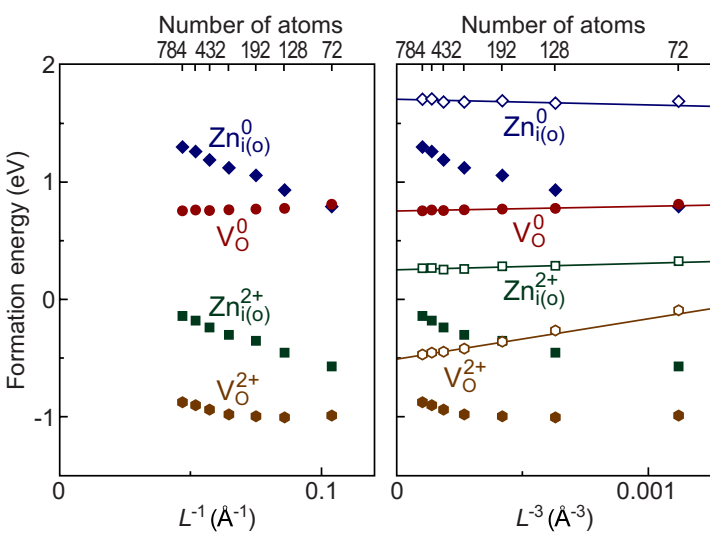

FIG. 1. (Color online) Formation energies of $V_{\mathrm{O}}$ and $\mathrm{Zn}_{i(o)}$ in the neutral and $2+$ charge states obtained by using the GGA as a function of $L^{-1}$ and $L^{-3}$ (where $L$ is the average interdefect distance). The energies are for the oxygen-poor limit and the Fermi energy at the valence-band maximum. The filled and open symbols denote the values before and after the Madelung energy corrections (Refs. 34 and 35).

For the finite-size corrections of the defect formation energies, the cell-size dependence was systematically investigated by using the GGA for all the considered defects. As typical examples, the results for $V_{\mathrm{O}}$ and $\mathrm{Zn}_{i(o)}$ in the neutral and $2+$ charge states are shown in Fig. 1. For charged defects, the $L^{-1}$ and $L^{-3}$ dependences of the formation energies are expected on the basis of the correction model by Leslie and Gillan, ${ }^{34}$ and by Makov and Payne. ${ }^{35}$ After subtracting the Madelung energies ( $L^{-1}$ dependences) estimated using $q_{c}=q$, i.e., formal defect charge, and $\epsilon_{0}=9.5$, where overestimation of the electronic dielectric constant by the GGA is taken into account, ${ }^{36,42}$ only nearly linear $L^{-3}$ dependences are left, as illustrated for $V_{\mathrm{O}}^{2+}$ and $\mathrm{Zn}_{i(o)}^{2+}$ in the right panel of Fig. 1. This demonstrates the appropriateness of the correction model and that the formation energies can be extrapolated to the dilute (infinite cell-size) limit using the $L^{-3}$ dependences obtained by a linear fit.

The neutral defects show a noteworthy behavior. The formation energy of $V_{\mathrm{O}}^{0}$ only slightly depends on the cell size, as expected from its neutral and localized nature. In contrast, $\mathrm{Zn}_{i(o)}^{0}$ shows a strong and nearly linear $L^{-1}$ dependence, as recognized in the left panel of Fig. 1. The slope is almost identical to that for $\mathrm{Zn}_{i(o)}^{2+}$, indicating the presence of a Madelung energy contribution with a similar magnitude. Indeed, a Madelung energy correction for $\mathrm{Zn}_{i(o)}^{0}$ using a charge of $q_{c}$ $=2$ leads to a good linear $L^{-3}$ dependence. As will be discussed later, the filling of conduction-band-like states with delocalized character, occurring also for $\mathrm{Zn}_{i(t)}, \mathrm{Zn}_{\mathrm{O}}, \mathrm{H}_{i}$, and $\mathrm{H}_{\mathrm{O}}$, does not effectively screen the charge of the point defect, leaving a long-range Madelung interaction between the point defects even in the neutral supercells. A similar behavior has been reported for the neutral defects in $\mathrm{InP}^{43}$ The proper charge $q_{c}$ for the Madelung energy corrections is, therefore, approximately given by the formal defect charge plus the number of delocalized conduction-band electrons $n_{e}$, $q_{c}=q+n_{e}$, or minus the number of delocalized valence-band holes $n_{h}, q_{c}=q-n_{h}$. As will be shown later for the examples of $V_{\mathrm{O}}$ and $\mathrm{Zn}_{i}$, these numbers are most easily determined by
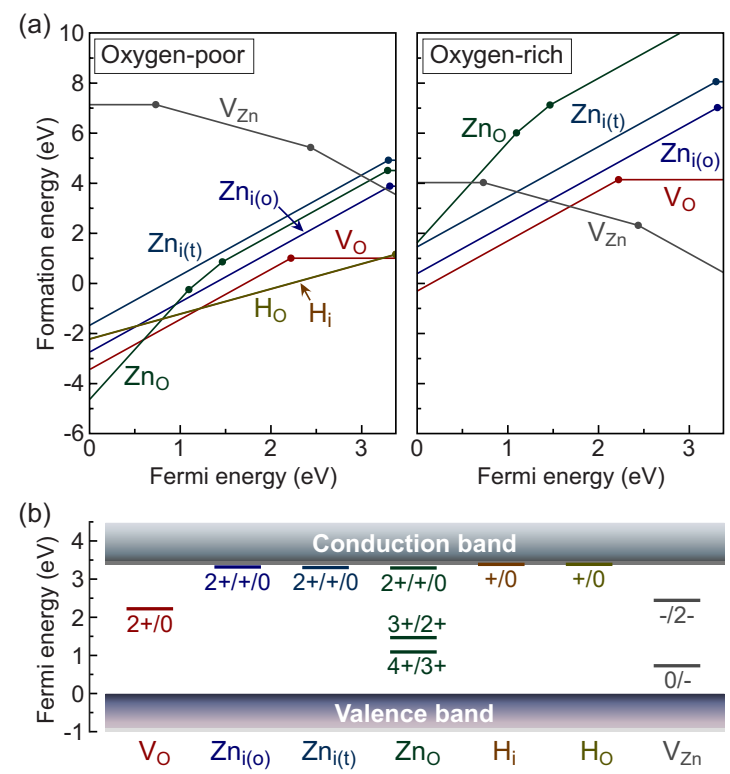

FIG. 2. (Color online) (a) Defect formation energies as a function of the Fermi energy at the oxygen-poor and oxygen-rich limits, which were obtained using the $\operatorname{HSE}(a=0.375)$ hybrid functional with the finite-size corrections. The slope corresponds to the charge state. (b) Defect transition levels equivalent to the filled circles in (a), alongside the relevant charge states.

inspecting the band structure: $n_{e}=2$ for $\mathrm{Zn}_{i}^{0}$ and $\mathrm{Zn}_{\mathrm{O}}^{0}, n_{e}=1$ for $\mathrm{Zn}_{i}^{+}, \mathrm{Zn}_{\mathrm{O}}^{+}, \mathrm{H}_{i}^{0}$, and $\mathrm{H}_{\mathrm{O}}^{0}$, and $n_{e} / n_{h}=0$ for the rest. To accurately predict the defect formation energies and transition levels, finite-size corrections including the Madelung contributions are, thus, also required for noncharged defects with extended defect states. This has not been done in any of the previous studies.

Figure 2(a) presents the formation energies obtained using the $\operatorname{HSE}(a=0.375)$ hybrid functional with the finite-size corrections, as a function of the Fermi energy. The range of the Fermi energy is given by the calculated valence-band maximum $(0 \mathrm{eV})$ and conduction-band minimum (CBM) (3.4 $\mathrm{eV})$. For each geometrical defect, only the charge states that are energetically most favorable at a given Fermi energy are shown. The Fermi energies at which the slopes change correspond to the positions of thermodynamic transition levels, which are also depicted in Fig. 2(b) together with the relevant charge states.

Among the native donorlike defects, $V_{\mathrm{O}}$ shows the lowest formation energy under most conditions. Notably, the formation energy for the neutral state $\left(V_{\mathrm{O}}^{0}\right)$ is only $1.0 \mathrm{eV}$ at the oxygen-poor limit. It exhibits a $2+/ 0$ transition level at 1.2 $\mathrm{eV}$ below the CBM, confirming the previously found negative- $U$ character, i.e., the instability of $V_{\mathrm{O}}^{+}{ }^{2,4-10,14-16}$ The zinc interstitials, $\mathrm{Zn}_{i(o)}$ and $\mathrm{Zn}_{i(t)}$, have transition levels located at $\sim 0.05$ and $\sim 0.1 \mathrm{eV}$ below the CBM, respectively. These levels are regarded here as transition levels among the three charge states, $2+1+/ 0$, because the $2+/+$ and $+/ 0$ transition levels are different only by less than $0.05 \mathrm{eV}$, which is comparable to the accuracy of the present calculations. The possession of the $2+/+/ 0$ transition levels near the $\mathrm{CBM}$ indicates that the zinc interstitials are single or double shallow donors, which is consistent with the experi- 


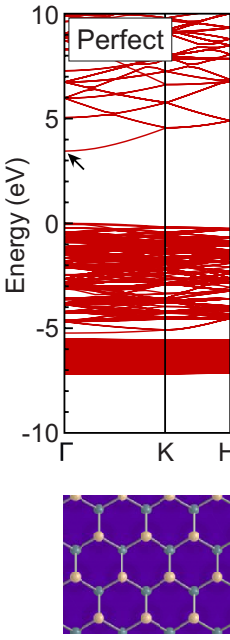

Occupancy: 0
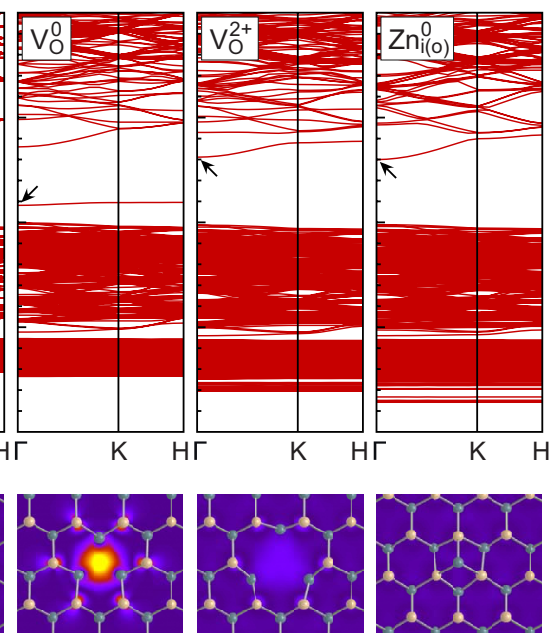

2

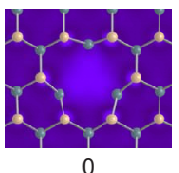

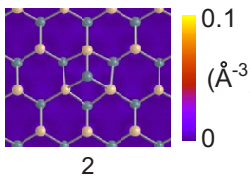

FIG. 3. (Color online) Band structure for the perfect crystal, $V_{\mathrm{O}}$ in the neutral and $2+$ charge states, and $\mathrm{Zn}_{i(o)}$ in the neutral charge state, which was obtained using the $\operatorname{HSE}(a=0.375)$ hybrid functional. Shown below are the squared wave functions of the states designated by the arrows, which are plotted for the middle of the (0001) $\mathrm{Zn}$ and $\mathrm{O}$ planes adjacent to the defects. The projected $\mathrm{Zn}$ and $\mathrm{O}$ atom positions are denoted with green (dark) and yellow (light) circles, respectively.

mental report (donor energy: $0.03 \mathrm{eV}) .{ }^{44}$ However, for the Fermi energy close to the CBM, the formation energies are as high as 4 and $5 \mathrm{eV}$ even at the oxygen-poor limit. Therefore, the zinc interstitials are unlikely to form with a substantial concentration in $n$-type $\mathrm{ZnO}$. $\mathrm{Zn}_{\mathrm{O}}$ also has a high formation energy under $n$-type conditions. In addition to the shallow $2+/+/ 0$ transition level, it shows $4+/ 3+$ and 3 $+/ 2+$ levels located below the middle of the band gap. Under oxygen-poor conditions and for a low Fermi energy, the formation energy is negative. This also holds for the other donorlike defects. A strong compensation of holes is therefore expected in $\mathrm{ZnO}$ grown under oxygen-poor conditions. For the hydrogen impurity, our results support its proposed role as a shallow donor. ${ }^{3,11}$ Both $\mathrm{H}_{i}$ and $\mathrm{H}_{\mathrm{O}}$ show $+/ 0$ transition levels nearly on the $\mathrm{CBM}$ and the formation energies are as low as that of $V_{\mathrm{O}}$ at the hydrogen-rich (oxygen-poor) limit.

Figure 3 shows the band structure for the 192-atom cells containing $V_{\mathrm{O}}^{0}, V_{\mathrm{O}}^{2+}$, and $\mathrm{Zn}_{i(o)}^{0}$ that were obtained using the $\operatorname{HSE}(a=0.375)$ hybrid functional, alongside the band structure for the perfect crystal. For $V_{\mathrm{O}}^{0}$, a localized occupied state is recognized in the band gap at $2.5-2.6 \mathrm{eV}$ below the CBM. It is accompanied by a significant inward relaxation of the first-nearest-neighbor $\mathrm{Zn}$ ions by $10 \%$, in stark contrast to the behavior of $V_{\mathrm{O}}^{2+}$ with an outward relaxation by $23 \%$, and no localized states in the band gap. $\mathrm{Zn}_{i(o)}^{0}$ leads only to small changes around the band gap. The highest occupied state is delocalized, which is comparable to the bottom of the conduction band in the perfect crystal. Due to this delocalized character, the removal of the electrons, i.e., changing the charge state to + or $2+$, does not alter the band structure. A similar character for the defect electronic state was also found by using the GGA. The defect state of $\mathrm{Zn}_{i(o)}^{0}$ is best described as a $\mathrm{Zn}^{2+}$ ion and two dispersed electrons located in the conduction band. If a finite-sized supercell is used to calculate the formation energy, the interaction energy between the ion and the two electrons in the conduction band is overestimated, and one can estimate the error by calculating the electrostatic interaction between +2 point charges and a homogeneous background with a total charge of -2 divided by the static dielectric constant. This is exactly the same correction usually applied for charged defects and it is easy to recognize that the combined correction is given by the Madelung energy of point charges $q_{c}$ in a homogeneous background charge, where $q_{c}$ is the formal defect charge plus the number of delocalized conduction-band electrons $n_{e}, q_{c}$ $=q+n_{e}$. This implies that for $\mathrm{Zn}_{i(o)}$, the same Madelung correction must be applied regardless of the formal charge state. A similar behavior in the band structure and the cell-size dependence was found for $\mathrm{Zn}_{i(t)}, \mathrm{Zn}_{\mathrm{O}}, \mathrm{H}_{i}$, and $\mathrm{H}_{\mathrm{O}}$.

The defect formation energies and transition levels determined in the present study not only show similarities but also important dissimilarities to the previous reports. ${ }^{2-17}$ (i) The thermodynamic transition level of $\mathrm{H}_{i}$ is predicted to be located just below the CBM in all the studies. (ii) The $2+/ 0$ thermodynamic transition level of $V_{\mathrm{O}}$ is estimated to be 1.2 $\mathrm{eV}$ below the CBM by using the present $\operatorname{HSE}(a=0.375)$ calculations. We obtained the same value using PBE0. An $\mathrm{LDA}+U$-based approach yields $1.0 \mathrm{eV},{ }^{9-11}$ whereas the uncorrected GGA value is $0.1 \mathrm{eV}$ with a large uncertainty due to the exceedingly small gap. The convergence to a single value of $\sim 1 \mathrm{eV}$ below the CBM for all methods that predict sufficiently large band gaps strongly supports the present value. This is in contrast to a posteriori band-gap corrections to the LDA/GGA that yield much deeper levels (e.g., $2.2 \mathrm{eV}$ reported in Ref. 8). (iii) Optical $V_{\mathrm{O}}$ transition levels: A recent B3LYP hybrid functional study provided a very deep transition level for $V_{\mathrm{O}}$, i.e., $3.0 \mathrm{eV}$ below the CBM. ${ }^{16}$ This level was determined using the one-electron energy of $V_{\mathrm{O}}^{0}$ at the $\Gamma$-point, and as shown in Fig. 3, the $\operatorname{HSE}(a=0.375)$ oneelectron energy at the $\Gamma$-point, i.e., $2.6 \mathrm{eV}$ below the CBM is close to this value. The main origin of the large difference between the one-electron energies and the thermodynamic transition energies is the huge relaxation energy of $4.2 \mathrm{eV}$ (Stokes shift), which happens when two electrons are removed from $V_{\mathrm{O}}^{0}$ to form $V_{\mathrm{O}}^{2+}$. In fact, the optical transition energy for exciting an electron from the $V_{\mathrm{O}}^{0}$ defect state to the $\mathrm{CBM}\left(V_{\mathrm{O}}^{0} \rightarrow V_{\mathrm{O}}^{+}+e^{-}\right)$, without ionic relaxation, is determined to be $2.6 \mathrm{eV}$, which is identical to the one-electron energy. This value is in reasonable agreement with the experimental onset for the creation of paramagnetic centers $(3.1 \mathrm{eV}) .{ }^{45}$

Concerning the formation energies, the correction schemes for the LDA/GGA show a huge spread, as mentioned for an example of $V_{\mathrm{O}}$ in the introduction. (i) The $V_{\mathrm{O}}$ formation energy of $1.0 \mathrm{eV}$ using $\operatorname{HSE}(a=0.375)$ at the oxygen-poor limit (0.9 eV using PBE0) is close to a straightforward GGA value and that with a posteriori band-gap corrections $^{8}$ (both $0.8 \mathrm{eV}$ ), whereas the LDA $+U$-based approach provides a much higher value $(3.7 \mathrm{eV}$ in Refs. 10 and 11). It is noted that our value of $\sim 1 \mathrm{eV}$ is low enough to account for the observed nonstoichiometry: e.g., 190 ppm for the specimen treated at $1373 \mathrm{~K} .^{46}$ (ii) The formation energies of $\mathrm{H}_{i}$ and $\mathrm{H}_{\mathrm{O}}$ at the hydrogen-rich limit, which are $1.2 \mathrm{eV}$ or 
lower depending on the Fermi energy, are also close to an experimental estimate of $0.8 \mathrm{eV},{ }^{47}$ again in contrast to the $\mathrm{LDA}+U$-based approach yielding 2.6 and $2.7 \mathrm{eV}$, respectively. ${ }^{11}$ (iii) Another consistency between our results and the experimental observation is found in the relative defect energetics: a strong preference for the donorlike defects over acceptorlike $V_{\mathrm{Zn}}$ under oxygen-poor conditions. This tendency is readily expected from the nonstoichiometric and $n$-type behavior of $\mathrm{ZnO}$ but has not been reproduced by the previous calculations other than applying a posteriori bandgap corrections to the LDA/GGA, as suggested in Refs. 4 and 8. Since $V_{\mathrm{Zn}}$ is not expected to exert significant effects on the composition and carrier concentration under oxygenpoor conditions, $V_{\mathrm{O}}$ (and also $\mathrm{H}_{\mathrm{O}}$, which can be regarded as a complex of $V_{\mathrm{O}}$ and $\mathrm{H}_{i}$ ) should dominantly contribute to nonstoichiometry, and the hydrogen impurities, $\mathrm{H}_{i}$ and $\mathrm{H}_{\mathrm{O}}$, and/or metastable $V_{\mathrm{O}}$ with a shallow donor state $^{7}$ can effectively act as donors. The present hybrid functional approach in conjunction with careful finite-size corrections for both charged and noncharged supercells, thus, provides defect energetics consistent with the relevant experimental observations.

\section{CONCLUSIONS}

The native defects and the hydrogen impurity in $\mathrm{ZnO}$ were investigated using first-principles calculations based on hybrid Hartree-Fock density functionals in conjunction with careful finite-size corrections. The results provide a clear picture of the defect energetics and electronic structure. Among the donorlike defects, $V_{\mathrm{O}}$ as a deep donor, and $\mathrm{H}_{i}$ and $\mathrm{H}_{\mathrm{O}}$ as shallow donors, are likely to form with a substantial concentration in $n$-type $\mathrm{ZnO}$. $\mathrm{Zn}_{i}$ and $\mathrm{Zn}_{\mathrm{O}}$ are shallow donors but their formation is energetically much less favorable. A strong preference for $V_{\mathrm{O}}, \mathrm{H}_{i}$, and $\mathrm{H}_{\mathrm{O}}$ over acceptorlike $V_{\mathrm{Zn}}$ is found under oxygen-poor conditions. It is therefore suggested that $V_{\mathrm{O}}$ (and also $\mathrm{H}_{\mathrm{O}}$ ) contributes to nonstoichiometry, and $\mathrm{H}_{i}$ and $\mathrm{H}_{\mathrm{O}}$ act as donors, both without significant compensation by $V_{\mathrm{Zn}}$. The present approach provides defect energetics in $\mathrm{ZnO}$ consistent with the relevant experimental observations without resorting to the empirical corrections for the valence and conduction-band positions. This constitutes a major step forward in modeling the complex behavior of semiconductors.

\section{ACKNOWLEDGMENTS}

Support by the Grant-in-Aid for Priority Area (No. 474) and Global COE Program from the MEXT, Japan, and the Austrian FWF is gratefully acknowledged. *oba@cms.mtl.kyoto-u.ac.jp

${ }^{1}$ D. C. Look, B. Claflin, Y. I. Alivov, and S. J. Park, Phys. Status Solidi A 201, 2203 (2004).

${ }^{2}$ A. F. Kohan, G. Ceder, D. Morgan, and Chris G. Van de Walle, Phys. Rev. B 61, 15019 (2000).

${ }^{3}$ C. G. Van de Walle, Phys. Rev. Lett. 85, 1012 (2000).

${ }^{4}$ S. B. Zhang, S.-H. Wei, and A. Zunger, Phys. Rev. B 63, 075205 (2001).

${ }^{5}$ F. Oba, S. R. Nishitani, S. Isotani, H. Adachi, and I. Tanaka, J. Appl. Phys. 90, 824 (2001); 90, 3665 (2001).

${ }^{6}$ E.-C. Lee, Y.-S. Kim, Y.-G. Jin, and K. J. Chang, Phys. Rev. B 64, 085120 (2001).

${ }^{7}$ S. Lany and A. Zunger, Phys. Rev. B 72, 035215 (2005).

${ }^{8}$ S. Lany and A. Zunger, Phys. Rev. Lett. 98, 045501 (2007).

${ }^{9}$ A. Janotti and C. G. Van de Walle, Appl. Phys. Lett. 87, 122102 (2005).

${ }^{10}$ A. Janotti and C. G. Van de Walle, Phys. Rev. B 76, 165202 (2007).

${ }^{11}$ A. Janotti and C. G. Van de Walle, Nat. Mater. 6, 44 (2007).

${ }^{12}$ M. G. Wardle, J. P. Goss, and P. R. Briddon, Phys. Rev. B 72, 155108 (2005).

${ }^{13}$ M. G. Wardle, J. P. Goss, and P. R. Briddon, Phys. Rev. Lett. 96, 205504 (2006).

${ }^{14}$ P. Erhart, K. Albe, and A. Klein, Phys. Rev. B 73, 205203 (2006).

${ }^{15}$ J.-L. Zhao, W. Zhang, X.-M. Li, J.-W. Feng, and X. Shi, J. Phys.: Condens. Matter 18, 1495 (2006).

${ }^{16}$ C. H. Patterson, Phys. Rev. B 74, 144432 (2006).

${ }^{17}$ A. A. Sokol, S. A. French, S. T. Bromley, C. R. A. Catlow, H. J. J. van Dam, and P. Sherwood, Faraday Discuss. 134, 267
(2007).

${ }^{18}$ S. F. J. Cox et al., Phys. Rev. Lett. 86, 2601 (2001).

${ }^{19}$ D. M. Hofmann, A. Hofstaetter, F. Leiter, H. J. Zhou, F. Henecker, B. K. Meyer, S. B. Orlinskii, J. Schmidt, and P. G. Baranov, Phys. Rev. Lett. 88, 045504 (2002).

${ }^{20}$ E. V. Lavrov, F. Borrnert, and J. Weber, Phys. Rev. B 72, $085212(2005)$

${ }^{21}$ G. A. Shi, M. Stavola, S. J. Pearton, M. Thieme, E. V. Lavrov, and J. Weber, Phys. Rev. B 72, 195211 (2005).

${ }^{22}$ D. C. Look, G. C. Farlow, P. Reunchan, S. Limpijumnong, S. B. Zhang, and K. Nordlund, Phys. Rev. Lett. 95, 225502 (2005).

${ }^{23}$ D. C. Reynolds, D. C. Look, B. Jogai, C. W. Litton, G. Cantwell, and W. C. Harsch, Phys. Rev. B 60, 2340 (1999).

${ }^{24}$ P. E. Blöchl, Phys. Rev. B 50, 17953 (1994).

${ }^{25}$ J. P. Perdew, K. Burke, and M. Ernzerhof, Phys. Rev. Lett. 77, 3865 (1996).

${ }^{26}$ J. P. Perdew, M. Ernzerhof, and K. Burke, J. Chem. Phys. 105, 9982 (1996).

${ }^{27}$ J. Heyd, G. E. Scuseria, and M. Ernzerhof, J. Chem. Phys. 118, 8207 (2003); 124, 219906 (2006)

${ }^{28}$ G. Kresse and J. Hafner, Phys. Rev. B 48, 13115 (1993).

${ }^{29}$ G. Kresse and J. Furthmüller, Phys. Rev. B 54, 11169 (1996).

${ }^{30}$ G. Kresse and D. Joubert, Phys. Rev. B 59, 1758 (1999).

${ }^{31}$ J. Paier, R. Hirschl, M. Marsman, and G. Kresse, J. Chem. Phys. 122, 234102 (2005).

${ }^{32}$ J. Paier, M. Marsman, K. Hummer, G. Kresse, I. C. Gerber, and J. G. Ángyán, J. Chem. Phys. 124, 154709 (2006); 125, 249901 (2006).

${ }^{33}$ C. G. Van de Walle and J. Neugebauer, J. Appl. Phys. 95, 3851 (2004). 
OBA et al.

${ }^{34}$ M. Leslie and M. J. Gillan, J. Phys. C 18, 973 (1985).

${ }^{35}$ G. Makov and M. C. Payne, Phys. Rev. B 51, 4014 (1995).

${ }^{36}$ N. Ashkenov et al., J. Appl. Phys. 93, 126 (2003).

${ }^{37}$ C. Persson, Y.-J. Zhao, S. Lany, and A. Zunger, Phys. Rev. B 72, 035211 (2005).

${ }^{38}$ J. Uddin and G. E. Scuseria, Phys. Rev. B 74, 245115 (2006).

${ }^{39}$ J. Albertsson, S. C. Abrahams, and A. Kvick, Acta Crystallogr., Sect. B: Struct. Sci. 45, 34 (1989).

${ }^{40}$ J. D. Cox, D. D. Wagman, and V. A. Medvedev, CODATA Key Values for Thermodynamics (Hemisphere, New York, 1989).

${ }^{41}$ W. Göpel, J. Pollmann, I. Ivanov, and B. Reihl, Phys. Rev. B 26, 3144 (1982).
${ }^{42}$ M. Shishkin and G. Kresse, Phys. Rev. B 75, 235102 (2007).

${ }^{43}$ C. W. M. Castleton and S. Mirbt, Phys. Rev. B 70, 195202 (2004).

${ }^{44}$ D. C. Look, J. W. Hemsky, and J. R. Sizelove, Phys. Rev. Lett. 82, 2552 (1999).

${ }^{45}$ F. Leiter, H. Alves, D. Pfisterer, N. G. Romanov, D. M. Hofmann, and B. K. Meyer, Physica B (Amsterdam) 340-342, 201 (2003).

${ }^{46}$ K. I. Hagemark and P. E. Toren, J. Electrochem. Soc. 122, 992 (1975).

${ }^{47}$ J. J. Lander, J. Phys. Chem. Solids 3, 87 (1957). 